\title{
Influência da estrutura urbana na decisão de realizar viagens a pé em Porto Alegre
}

\author{
Ana Margarita Larranaga ${ }^{1}$, Helena Beatriz Bettella Cybis ${ }^{2}$ e Tânia Batistela Torres ${ }^{3}$
}

\begin{abstract}
Resumo: Estudos realizados em países desenvolvidos revelam que mudanças nas características da estrutura urbana podem ter impacto significativo sobre o padrão de viagens. Este artigo visa: (i) testar se estas relações reportadas na literatura sobre a relação entre a estrutura urbana e comportamento de viagem são mantidas numa cidade como Porto Alegre; (ii) testar se a magnitude do impacto destas características se mantém; e (iii) comparar o impacto na decisão de caminhar em relação aos resultados obtidos num estudo anterior para a frequência de viagens a pé. Modelos logit binomial e logit mistos foram estimados para modelar a decisão de caminhar. Elasticidades da decisão de caminhar com relação às variáveis da estrutura urbana foram calculadas. Os resultados mostram que, em Porto Alegre, a estrutura urbana influencia a decisão de realizar viagens a pé. No entanto, existem diferenças nas magnitudes do impacto de algumas variáveis. As elasticidades calculadas para a decisão de caminhar com relação às variáveis da estrutura urbana foram maiores do que em outros estudos. Valores de elasticidades podem ser usados em previsões de modelos de demanda.
\end{abstract}

Palavras-chave: estrutura urbana, modelos de escolha discreta, viagens a pé.

\begin{abstract}
Studies conducted in cities from developed countries reveal that changes in the built environment can have significant impact on travel behavior. This article aims to: (i) test whether these relationships reported in the literature between built environment and travel behavior hold in a city like Porto Alegre; (ii) test whether the magnitude of the impact of these characteristics also hold; and (iii) compare the impact force in the decision to walk with the impact on frequency of walking, which was modeled in a previous study. Binomial logit and mixed logit models were estimated to model the decision to walk. Elasticities of the decision to walk for the built environment characteristics were computed. The results show that, in Porto Alegre, the built environment influences the decision to walk. However, there are differences in the magnitude of the impact of certain variables. The elasticities computed for the decision to walk with respect to the variables of the built environment were higher than in other studies. Values elasticities can be used in forecasting demand models.
\end{abstract}

Keywords: built environment, discrete choice models, walking.

\section{INTRODUÇÃO}

Análises sobre a influência das características urbanas no padrão de viagens podem ser observadas desde o início do século 20. Na década de 1990, com o crescente interesse em correntes como "Novo Urbanismo" (New Urbanism), "Crescimento Inteligente" (Smart Growth) e "Desenvolvimento Orientado pelo Trânsito" (Transit Oriented Development), o número de estudos aumentou exponencialmente (Zegras, 2010). Na maioria dos estudos, o padrão de viagens (representado pela frequência de viagens, modo, distância, duração, destino ou propriedade de automóvel) foi relacionado com características da estrutura urbana representadas por três componentes principais: (i) padrão de uso do solo; (ii) desenho urbano; e (iii) sistema de transporte. Padrão de uso do solo refere-se à distribuição das atividades no espaço, incluindo a localização e densidade de diferentes atividades, tais como residencial, comercial, industrial. O desenho urbano significa o desenho da cidade e os elementos físicos dentro dele, incluindo tanto sua organização como sua aparência. Finalmente, o sistema de transporte compreende a infraestrutura física das vias, calçadas,

\footnotetext{
${ }^{1}$ Ana Margarita Larranaga, Laboratório de Sistemas de Transportes LASTRAN, UFRGS. (analarra@producao.ufrgs.br)

2 Helena Beatriz Bettella Cybis, Laboratório de Sistemas de Transportes LASTRAN, UFRGS. (helenabc@ufrgs.br)

${ }^{3}$ Tânia Batistela Torres, Laboratório de Sistemas de Transportes -

LASTRAN, UFRGS. (taniabatistela@gmail.com)
}

Manuscrito recebido em 20/05/2015 e aprovado para publicação em 17/09/2015.

Este artigo é parte de TRANSPORTES v. 23, n. 4, 2015. ISSN: 2237-1346

(Online). DOI:10.14295/transportes.v23i4.924 ciclovias, vias férreas, pontes etc., bem como as características de serviço prestado, conforme determinado pelos níveis de tráfego, frequências de ônibus e similares (Handy et al., 2002).

Atualmente, existe um crescente reconhecimento que mudanças nas características da estrutura urbana podem ter impacto significativo sobre o padrão de viagens. Pesquisas realizadas (Baran et al., 2008; Cao et al., 2006; Cervero e Duncan, 2003; Ewing e Cervero, 2001, 2010; Frank e Engelke, 2001; Lee e Moudon, 2006) sugerem que pessoas que vivem em bairros orientados para pedestres, caracterizados por uso de solo misto, alta conectividade viária e alta densidade populacional, são estimulados a dirigir menos e utilizar outros modos de transporte, como transporte público, bicicleta e a pé.

No entanto, depois destes anos de pesquisa e análises, ainda restam dúvidas sobre o impacto quantificável do ambiente construído sobre o padrão de viagens e pesquisas nesta área continuam. Tentativas de generalizar os resultados, sob a forma de elasticidades de frequências de viagens e distâncias percorridas pelos veículos em relação a diferentes medidas da estrutura urbana, foram realizados por Ewing e Cervero (2001, 2010). Apesar destes esforços, generalizar a partir desta pesquisa continua sendo um desafio, devido a variações na: (i) escala de análise (nível "meso" ou "micro"); (ii) tipos de medidas da estrutura urbana utilizadas (densidade populacional, densidade residencial, medidas de entropia para o uso do solo etc.); (iii) dados de viagens utilizados (agregadas ou desagregadas, individual ou familiar); (iv) abordagens analíticas e variáveis de controle utilizadas; e (v) resultados finais medidos (frequências de 
viagem, escolha de modo, distâncias percorridas etc.) (Zegras, 2010).

A grande parte destes estudos foi desenvolvida em países industrializados. No entanto a influência da estrutura urbana no padrão de viagens em cidades em desenvolvimento pode ser essencial para orientar projetos urbanísticos e de transportes que visem preservar o ambiente de forma sustentável. Infelizmente, pesquisas em cidades em desenvolvimento continuam sendo limitadas.

Estudos nesta linha de pesquisa na América do Sul são, por exemplo, os realizados nas cidades de Bogotá (Cervero et al., 2009) e Santiago (Zegras, 2004, 2010). Cervero et al. (2009) analisaram a relação entre estrutura urbana e viagens utilitárias a pé e de bicicleta. Zegras (2004) estudou a interação entre viagens a pé e estrutura urbana para a cidade de Santiago, e em outro trabalho posterior (Zegras, 2010) pesquisou a relação entre estrutura urbana e número de automóveis no domicílio na mesma cidade. No Brasil, alguns estudos nesta linha foram realizados por Amâncio (2005); Fernandes et al. (2008), Deus (2008) e Larranaga et al. (2014). Amâncio (2005), pesquisou a existência de uma relação entre forma urbana e escolha a do modo a pé para a cidade de São Carlos-SP. Fernandes et al. (2008) analisaram a relação entre a forma urbana e o transporte no Município de Olinda-PE. Deus (2008) estudou a influência da estrutura urbana na escolha modal na cidade de UberlândiaMG. No entanto, estes estudos foram concebidos com objetivos específicos diferentes. Fernandes et al. (2008) estudaram unicamente as viagens pendulares e realizaram uma análise descritiva através de tabelas de frequências, Amâncio (2005) e Deus (2008) estimaram modelos genéricos, sem estratificação em relação ao motivo das viagens. Ainda, nenhum destes trabalhos mencionados, tanto no Brasil quanto nas outras cidades de América do Sul, incluíram as preferências de viagem. Preferências de viagem se referem à predisposição a usar um modo de transporte. Embora estes estudos contribuam substancialmente para o desenvolvimento desta linha de pesquisa, é evidente a necessidade de maiores pesquisas para aprofundar o tema e poder comparar os resultados obtidos. O estudo realizado por Larranaga et al. (2014) analisou o impacto da estrutura urbana na frequência de viagens a pé para a cidade de Porto Alegre (Brasil), considerando as preferências de viagem dos indivíduos além de características urbanas e socioeconômicas. O presente trabalho continua esse estudo, utilizando outra abordagem de análise e comparando os resultados com os obtidos nesse estudo.

Os estudos reportados na literatura (sintetizados por Ewing e Cervero; 2010) mostram que o impacto das variáveis da estrutura urbana pode ser diferente dependendo da variável dependente medida (frequência de viagens, escolha de modo, distâncias percorridas etc). Os resultados obtidos para Estados Unidos indicam que frequência de viagens é primeiramente dependente das características socioeconômicas dos indivíduos e em segundo lugar, dependente das características urbanas. Entretanto, a escolha do modo a pé depende de ambos, com intensidade semelhante (Ewing e Cervero; 2010). A determinação do impacto real de mudanças na estrutura urbana no comportamento de viagens é de vital importância para obter medidas eficazes e ganhos significativos com estratégias deste tipo.
Este artigo visa três objetivos. Primeiro, testar se a maioria das descobertas sobre a relação entre a estrutura urbana e comportamento de viagem reportadas na literatura são mantidas numa cidade de um país em desenvolvimento, Porto Alegre (Brasil) e, como tal, se eles são relevantes para a elaboração de políticas. A priori, espera-se replicar muitos dos resultados já relatados na literatura.

Em segundo lugar, no caso de verificar a hipótese do primeiro objetivo, objetiva-se testar se a magnitude do impacto destas características urbanas, reportadas na literatura para outras cidades, se mantém para Porto Alegre. Em terceiro lugar, comparar o impacto de variáveis associadas às características da estrutura urbana e socioeconômicas na decisão de caminhar com resultados obtidos em estudo anterior (Larranaga et al., 2014) para a frequência de viagens a pé. A magnitude do impacto na modelagem de "quanto as pessoas caminham" pode ser diferente do impacto na modelagem de "se as pessoas caminham ou não". Aplicações destes resultados, como por exemplo previsões de demanda - baseados em modelos de quatro etapas - ao variar características da estrutura urbana ou outras aplicações que busquem quantificar o impacto do ambiente construído (como proposto por Ewing e Cervero, 2010), precisam ter valores que reflitam o impacto real de cada variável urbana.

O restante deste artigo está organizado da seguinte forma. A Seção 2 descreve a metodologia adotada para a determinação das variáveis e formulação das modelos, a Seção 3 discute os dados coletados, e a Seção 4 apresenta os resultados da estimação. O trabalho finaliza com as conclusões e sugestões para futuras pesquisas.

\section{METODOLOGIA}

Esta seção descreve as variáveis da estrutura urbana consideradas e a abordagem adotada na modelagem.

\subsection{Variáveis}

A influência da estrutura urbana no padrão de viagem foi analisada através do modelo "5Ds".

Nesse modelo, as características da estrutura urbana são representadas por características principais ou dimensões que começam em inglês com a letra " $D$ ". Originalmente, se expressava a estrutura urbana através de três dimensões, propostas por Cervero e Kockelman (1997), conhecidas como "3Ds" (density, diversity, design): densidade, diversidade de uso do solo, desenho urbano. Posteriormente, foram agregadas duas dimensões (Ewing et al., 2009) formando as "5Ds" (density, diversity, design, distance to transit, destination accesibility): densidade, diversidade de uso do solo, desenho urbano, distância até o transporte público e acessibilidade ao destino.

Densidade refere-se à intensidade do uso do solo para habitação, emprego, e outras finalidades. Medidas de densidade são mensuradas em relação a uma área (unidade de área) líquida ou bruta. A intensidade de uso do solo pode ser indicada por variáveis que quantificam população, unidades habitacionais, emprego e área útil construída, ou por densidade global, que é medida pela combinação de população e emprego (Ewing e Cervero, 2010). 
Tabela 1. Variáveis da estrutura urbana do bairro de residência (500m)

\begin{tabular}{|c|c|c|}
\hline Dimensão & Variáveis Candidatas & Fonte de dados \\
\hline Densidade & $\begin{array}{l}\text { Densidade de Domicílios }\left(\mathrm{N}^{\circ} \text { de domicílios por }\right. \\
\left.\mathrm{km}^{2}\right) \\
\text { Densidade Populacional }\left(\mathrm{N}^{\circ} \text { de habitantes por } \mathrm{km}^{2}\right)\end{array}$ & IBGE (Instituto Brasileiro de Geografia e Estatística) \\
\hline $\begin{array}{l}\text { Diversidade de uso do } \\
\text { solo }\end{array}$ & $\begin{array}{l}\text { Entropia (escala entre } 0 \text { e } 1,1=\text { uso misto em per- } \\
\text { centuais iguais, } 0=\text { uso homogêneo) }\end{array}$ & $\begin{array}{l}\text { PMPA-IPTU (Prefeitura Municipal de Porto Alegre - } \\
\text { Imposto Predial e Territorial Urbano) }\end{array}$ \\
\hline Desenho urbano & $\begin{array}{l}\text { \% de Interseções } 4 \text { aproximações (em “cruz”) } \\
\text { Comprimento Médio da Quadra (m) } \\
\text { Declividade } \\
N^{o} \text { Acidentes por ano (totais e envolvendo pedes- } \\
\text { tres) }\end{array}$ & $\begin{array}{l}\text { PROCEMPA } \\
\text { EPTC (Companhia de Processamento de Dados do } \\
\text { Município de Porto Alegre - Empresa Pública de } \\
\text { Transporte e Circulação) }\end{array}$ \\
\hline $\begin{array}{l}\text { Disponibilidade de } \\
\text { transporte público }\end{array}$ & $\begin{array}{l}\text { Frequência: } \mathrm{n}^{\circ} \text { total de viagens de cada linha que } \\
\text { serve a área } \\
\text { Cobertura: } \sum \mathrm{n}^{\circ} \text { de pontos de parada de ônibus de } \\
\text { cada linha que serve a área /Superfície da área } \\
\text { Capacidade: } \sum \mathrm{n}^{\circ} \text { de lugares por linha* distância } \\
\text { percorrida pelo ônibus na área/ população total da } \\
\text { área. }\end{array}$ & EPTC (Empresa Pública de Transporte e Circulação) \\
\hline $\begin{array}{l}\text { Acessibilidade no des- } \\
\text { tino }\end{array}$ & $\begin{array}{l}\text { Densidade de Comércios e Serviços (Noestabeleci- } \\
\left.\text { mentos } / \mathrm{km}^{2}\right)\end{array}$ & $\begin{array}{l}\text { PMPA -SMIC ((Prefeitura Municipal de Porto Alegre } \\
\text { - Secretaria Municipal da Produção, Indústria e Comér- } \\
\text { cio) }\end{array}$ \\
\hline
\end{tabular}

A diversidade de uso do solo se refere às diferentes formas de utilização do uso do solo e aos níveis de representatividade do mesmo em uma determinada área (Ewing e Cervero, 2010). Com o objetivo de medir a diversidade foi proposto por Cervero e Kockelman (1997) o conceito de entropia. Uma baixa entropia significa que um determinado uso de solo é predominante na região. Bairros que possuem alta diversidade de uso do solo aproximam as origens e destinos, incentivando que viagens sejam realizadas a pé.

Desenho urbano refere-se à qualidade do meio ambiente para caminhadas e à configuração física das redes viárias. As redes viárias podem apresentar malhas urbanas densas com ruas retas, bem conectadas, ou outras configurações, que em um extremo oposto, apresentam redes curvas, ruas sem saída e poucas conexões (Ewing e Cervero, 2010). A primeira é uma estrutura que apresenta alta conectividade e torna o desenho urbano um fator incentivador de viagens a pé. Variáveis normalmente utilizadas para mensurar esta característica, são geralmente o "tamanho médio das quadras" e a "proporção de interseções com 4 aproximações".

Distância ao transporte público reflete a acessibilidade ao transporte público. Acessibilidade ao destino da viagem refere-se ao acesso, de todas as parcelas da população, a centros atratores de viagens (emprego, educação, saúde, compras). A Tabela 1 apresenta as variáveis da estrutura urbana adotadas para representar cada dimensão e as fontes de obtenção dos dados utilizados neste trabalho.

As características da estrutura urbana dos bairros foram obtidas pelo processamento de dados por SIG (Sistema de Informação Geográfica). As características foram medidas considerando uma área circular (buffer-ring) de $500 \mathrm{~m}$ de raio em torno dos centroides de cada um dos setores censitários selecionados no qual o domicílio entrevistado está inserido.

\subsection{Modelagem}

Modelos de escolha discreta foram estimados para determinar o efeito da estrutura urbana na decisão de caminhar. Os modelos de escolha discreta utilizados na modelagem da demanda de transportes estão construídos conforme a teoria da utilidade aleatória (McFadden, 1974). Baseiamse no princípio da maximização da utilidade, no qual o tomador de decisão é modelado selecionando a alternativa de maior utilidade dentre aquelas disponíveis no momento da escolha. $\mathrm{O}$ analista não possui informação completa sobre os elementos que cada indivíduo considera ao realizar sua escolha. Portanto, as utilidades são tratadas pelo analista como variáveis aleatórias (Ben-Akiva e Lerman, 1985). Assim, a utilidade aleatória de uma alternativa é expressa como a soma dos componentes observáveis ou sistemáticos (denotado como $\mathrm{V}_{\mathrm{iq}}$ ) e componentes não observáveis (denotado como $\varepsilon_{\mathrm{iq}}$ ) conforme Equação 1 (Domencich e McFadden, 1972).

$$
U_{i q}=V_{i q}+\varepsilon_{i q}
$$

O componente aleatório é necessário para capturar deficiências na especificação de atributos não observados, erros de medição, diferenças entre indivíduos, percepções incorretas de atributos e aleatoriedade inerente à natureza humana (Manski, 1977). Especificar o modelo requer a especificação do $\mathrm{V}_{\text {in }}$ e suposições sobre a distribuição conjunta da componente aleatória $\varepsilon_{\text {iq }}$. Diferentes hipóteses sobre a distribuição de probabilidade adotada para a componente aleatória $\varepsilon_{\mathrm{iq}}$ leva a diversos modelos de escolha discreta (Ortúzar e Willumsen, 2011; McFadden e Train, 2000).

O logit multinomial (multinomial logit - MNL) (McFadden, 1974) é um dos modelos mais simples de escolha discreta e também o mais utilizado. Ele se baseia na hi- 
pótese que o termo aleatório $\varepsilon_{\text {iq }}$ da função utilidade é identicamente e independentemente distribuído conforme uma distribuição de Gumbel (Valor Extremo tipo II). Este pressuposto para a distribuição dos resíduos é bastante simplista, uma vez que dependem da hipótese de independência e homocedasticidade dos resíduos (Ben-Akiva et al., 2003). Uma série de modelos com estruturas mais flexíveis têm sido propostos. Nos últimos anos, a utilização de modelos logit mistos, mixed logit- ML (Ben Akiva e Bolduc, 1996; Brownstone e Train, 1999), é cada vez maior, permitindo incorporar variações das preferências não observáveis. Estes modelos supõem um termo aleatório identicamente e independentemente distribuído conforme uma distribuição de Gumbel, assim como o MNL, mas com um componente aleatório adicional que permite trabalhar com maior flexibilidade. Dependendo dos pressupostos considerados sobre os diversos termos aleatórios é possível modelar correlação e heterocedasticidade (Brownstone e Train, 1999).

No presente trabalho, a decisão de realizar viagens a pé foi representada por uma variável binária, a qual adota o valor 1 quando o indivíduo realizou alguma viagem a pé no entorno da residência e o valor 0 no caso contrário. A decisão de caminhar foi modelada com dois tipos de estruturas: (i) logit binomial, o qual é um caso particular do logit multinomial quando existem unicamente duas alternativas; e (ii) logit mistos de componentes de erro (error components$E C$ ), para representar a possível correlação entre indivíduos do mesmo domicílio. A estimação dos modelos mistos de componente de erro foi adotada considerando que as viagens realizadas por diferentes residentes do mesmo domicílio podem estar correlacionadas. Por exemplo, uma viagem ao supermercado pode ser realizada por um dos residentes do domicílio, evitando a viagem do outro residente para satisfazer este motivo. Os modelos logit mistos permitem incluir um termo adicional que represente a correlação entre as viagens do mesmo domicílio.

As estimações foram realizadas com o software Biogeme (Bierlaire, 2003). Em todas as estruturas foram utilizadas funções de utilidade lineares nos parâmetros, prática usual na modelagem de demanda de transporte (Ben Akiva e Lerman, 1985).

Para comparar a importância das variáveis explicativas com diferentes escalas, foi calculada a elasticidade da probabilidade da decisão de caminhar em relação às variáveis explicativas. A elasticidade da probabilidade de escolha (decisão de caminhar) mede a sensibilidade da escolha dos indivíduos em relação a uma variável explicativa. Indica qual a mudança percentual na probabilidade do indivíduo escolher uma alternativa em função de uma mudança marginal no valor da variável explicativa da função utilidade (Ortúzar e Willumsen, 2011). O cálculo da elasticidade utilizou a equação proposta por Ortúzar e Willumsen (2011) (Equação 2) e posteriormente foram agregadas utilizando o método de enumeração amostral.

$$
E_{\text {PiqXikq }}=\theta_{i k} \cdot X_{i k q}\left(1-P_{i q}\right)
$$

em que $E_{P i q, X i k q}$ : é a elasticidade da probabilidade de escolha da alternativa $A_{i}$ em relação a uma mudança marginal de um determinado atributo $X_{i k q}$ para o indivíduo $q ; \theta_{i k}$ : valor do coeficiente do atributo $X_{i k}$ na opção de troca e $P_{i q}$ : probabilidade de escolha da alternativa $A_{i}$

\section{DADOS}

Este trabalho utilizou dados de um estudo realizado pelo Laboratório de Sistemas de Transportes (Lastran) da Universidade Federal do Rio Grande do Sul (UFRGS) descritos em Larrañaga (2012) e Larrañaga et al. (2014). A seguir são apresentados os dados, caracterizando a amostra e apresentando a estatística descritiva das variáveis utilizadas.

\subsection{Descrição dos dados}

Os dados do estudo consistem em informações de viagens, atitudinais, socioeconômicas e da estrutura urbana do bairro de residência. Os dados sobre viagens e alguns dos dados socioeconômicos e atitudinais foram provenientes de uma pesquisa domiciliar realizada em Porto Alegre especificamente para o estudo realizado no Lastran (Larranaga, 2012; Larranaga et al, 2014). Os dados da estrutura urbana foram obtidos de diferentes fontes e processados através de Sistema de Informação Geográfica (SIG).

A pesquisa domiciliar coletou informações sobre 884 indivíduos selecionados através de um método de amostragem em dois estágios: setores censitários e domicílios. Foram pesquisados 442 domicílios. As informações de viagens se referiam a todos os deslocamentos realizados pelos respondentes no dia anterior, inclusive deslocamentos curtos não medidos em pesquisas tradicionais. As características socioeconômicas medidas foram: idade, sexo do entrevistado, tipo de moradia, número de residentes no domicílio, número de automóveis no domicílio, rendimento familiar mensal, grau de instrução, posse de carteira de habilitação, ocupação prinicpal, e local de trabalho (fora ou em casa).

As características atitudinais foram avaliadas solicitando que os entrevistados indicassem o grau de concordância com um conjunto de afirmações. Os questionários continham doze afirmações referentes a preferências de viagem e percepção de segurança do bairro. Cada entrevistado deveria indicar seu grau de concordância com cada afirmação utilizando uma escala continua entre zero, "discordo completamente", e dez, "concordo completamente". A escala adotada corresponde à Escala Visual Analógica (EVA) (Wewers e Lowe, 1990). As primeiras nove afirmações procuravam traduzir um conceito geral de preferência ou predisposição à escolha de um modo de viagem. As últimas três procuravam identificar a percepção de segurança pública e de tráfego sobre o bairro. As características atitudinais e de percepção do bairro foram agrupadas por Larranaga et al. (2014) em três variáveis latentes através de um modelo de Múltiplas Causas e Múltiplos Indicadores (MIMIC). As três variáveis latentes extraídas foram: (i) Pro_caminhada, que indica uma predisposição a utilização do modo a pé ou transporte público nos deslocamentos diários; (ii) Pro_automóvel, indica uma predisposição à utilização do automóvel; (iii) Segurança_bairro, refere-se a percepção de segurança pública e de tráfego na proximidade da residência.

$\mathrm{O}$ presente artigo aborda unicamente a modelagem comportamental, utilizando as variáveis atitudinais estimadas no estudo de Larranaga et al. (2014) como variáveis explicativas. Por maiores detalhes sobre a estimação destas 


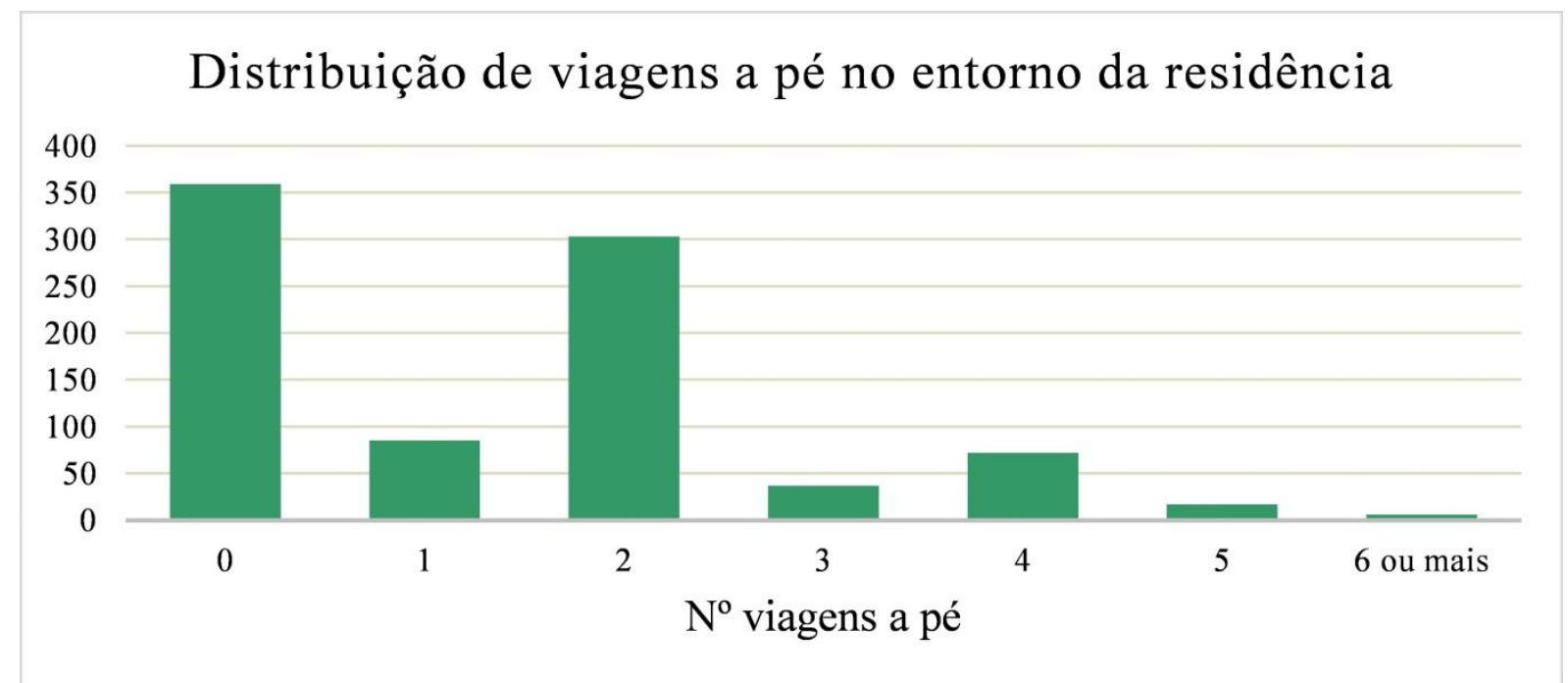

Figura 1. Viagens a pé por dia no entorno da residência

variáveis e/ou sobre os dados coletados consultar o trabalho referenciado.

\subsection{Caracterização das viagens}

A análise realizada neste estudo considera as viagens realizadas exclusivamente a pé. Não são considerados os deslocamentos a pé para acessar a outro modo de transporte. $\mathrm{Na}$ amostra pesquisada, 59\% dos entrevistados caminharam próximo à residência. Entretanto, apenas $34 \%$ realizaram o deslocamento a pé entre a origem e o destino final. A figura 1 apresenta a distribuição de viagens a pé no entorno da residência.

$\mathrm{Na}$ amostra pesquisada foi observado que os homens realizam, em média, mais viagens que as mulheres considerando todos os modos. Entretanto, as mulheres realizam mais viagens a pé do que os homens. A relação entre viagens e renda familiar mostrou que indivíduos de maior renda realizam mais viagens totais (considerando todos os modos). No entanto, realizam menos viagens a pé. Ainda, essas viagens são, fortemente, limitadas pela distância a ser percorrida e o horário, $79 \%$ dos entrevistados caminhou até 1500 metros, e apenas $2 \%$ dessas foram realizadas a noite.

\subsection{Estatística descritiva}

A Tabela 2 apresenta a estatística descritiva das variáveis dependentes e independentes utilizadas nos modelos finais de escolha discreta.

\section{RESULTADOS}

Os resultados dos modelos logit binomial e logit misto são apresentados na Tabela 3.

Conforme mostra a Tabela 3, o teste da razão de verossimilhança com $10 \%$ de significância revela que a adição do componente de erro nos modelos mistos ao modelo MNL tem um impacto significativo (valor-p Sigma=0,10). Desta forma, podemos afirmar que os dados de indivíduos de um mesmo domicílio estão correlacionados, sendo adequado o uso do modelo logit mistos na estimação.

$\mathrm{O}$ ajuste geral foi avaliado através do Pseudo- $\mathrm{R}^{2}$, o qual varia entre 0 e 1 (aderência total aos dados). $\mathrm{O}$ valor obtido para esse parâmetro é aceitável, similar ao encontrado em outros estudos (Cao et al., 2006; Sehatzadeh et al.,
2011). Valores próximos a 0,4 são considerados excelentes (Ortúzar e Willumsen, 2011). Quatro variáveis da estrutura urbana foram preditores significativos: Densidade Populacional, Densidade de Interseções 4 aproximações, Declividade e Densidade de comércios.

Bairros com alta densidade populacional e alta densidade de comércios e serviços permitem concentrar e aproximar origens e destinos de viagens, brindando mais oportunidades para deixar o automóvel em casa e caminhar até o destino. Pela natureza do cálculo, geralmente se utilizam dimensões separadas para caracterizar a densidade na origem, densidade populacional ou residencial, e no destino, densidade de comércios, empregos ou outras oportunidades de destinos de viagem. Mas é a relação sinérgica entre as duas que afeta o padrão de viagens. Conjuntos de vivendas, condomínios, ou conjuntos habitacionais localizados em área suburbana, por exemplo, longe de serviços básicos, podem apresentar densidades populacionais ou residenciais altas. Entretanto, isto não reduz a necessidade de utilizar modos motorizados ou viajar distâncias consideráveis para realizar atividades diárias. Os modelos apresentados captaram essa integração, mostrando que valores altos de densidade populacional e de comércios e serviços estimulam os deslocamentos a pé. Esse efeito positivo da densidade na promoção de viagens a pé coincide com os resultados encontrados em outros estudos, como os realizados por Larrañaga et al. (2014); Boarnet et al. (2008), Chatman (2009), Ewing et al.(2009), Frank et al.(2009), Naess (2005).

A variável Densidade de Interseções 4 aproximações foi calculada como a relação entre o número de interseções em "cruz" e o número de interseções totais numa área circular de $500 \mathrm{~m}$ (com origem no centro geométrico do setor censitário de residência). Esta é uma medida frequentemente utilizada (Boarnet et al., 2008; Ewing et al., 2009) para indicar a configuração viária, a qual pode ser em forma de grelha, ou apresentar configuração com curvas. Padrões viários em forma de grelha são representados por um número alto de interseções em "cruz", em detrimento de interseções em T ou cul-de-sacs. Os modelos apresentados mostraram que configurações viárias em forma de grelha, com muitas interseções, estimulam os deslocamentos a pé, pois aumentam a conectividade, oferecendo maiores oportunidades de rotas entre origem e destino. Esse resultado coincide 
Tabela 2. Estatística descritiva das variáveis

\begin{tabular}{|c|c|c|c|c|}
\hline & Média & $\begin{array}{l}\text { Desvio } \\
\text { Padrão }\end{array}$ & Mínimo & Máximo \\
\hline \multicolumn{5}{|l|}{ Variável dependente } \\
\hline Decisão de caminhar (1: caminha; 0: não caminha.) & 0,35 & 0,48 & 0,00 & 1,00 \\
\hline \multicolumn{5}{|l|}{ Socioeconômicas } \\
\hline Idade (anos) & 43 & 17,7 & 0,00 & 1,00 \\
\hline Gênero (1:masculino, 0:feminino) & 0,45 & 0,5 & 0,00 & 1,00 \\
\hline Grau de Instrução Baixo (1: não alfabetizado, 0:outro) & 0,03 & 0,17 & 0,00 & 1,00 \\
\hline Grau de Instrução Médio (1: ensino fundamental ou médio com/incom, 0:outro) & 0,58 & 0,49 & 0,00 & 1,00 \\
\hline Grau de Instrução Alto (1: superior ou pós-graduação com/incom, 0: outro) & 0,39 & 0,49 & 0,00 & 1,00 \\
\hline Disponibilidade de automóvel $(1,0)$ & 0,43 & 0,49 & 0,00 & 1,00 \\
\hline Local de trabalho (1:outro; $0:$ casa) & 0,68 & 0,32 & 0,00 & 1,00 \\
\hline Número de automóveis no domicílio (nº.) & 0,83 & 0,75 & 0,00 & 1,00 \\
\hline Renda baixa $(1:<\mathrm{R} \$ 3300,0$ : outro $)$ & 0,36 & 0,48 & 0,00 & 1,00 \\
\hline Renda média (1: 3300 a $\mathrm{R} \$ 6500,0$ : outro) & 0,24 & 0,42 & 0,00 & 1,00 \\
\hline Renda alta $(1:>\mathrm{R} \$ \$ 6500,0$ : outro $)$ & 0,4 & 0,49 & 0,00 & 1,00 \\
\hline \multicolumn{5}{|l|}{ Estrutura Urbana } \\
\hline Densidade populacional $\left(\mathrm{n}^{\circ}\right.$ habitantes $/ \mathrm{km}^{2}$ ), círculo $500 \mathrm{~m}$ & 12807,1 & 5813,37 & 3400,98 & 26705,04 \\
\hline$\%$ interseções com 4 aproximações, círculo $500 \mathrm{~m}$ & 35 & 15 & 0,08 & 0,62 \\
\hline Declividade media (\%), círculo $500 \mathrm{~m}$ & 3,5 & 2,7 & 0,45 & 10,68 \\
\hline Comprimento de quadra, círculo $500 \mathrm{~m}(\mathrm{~m})$ & 95,07 & 19,36 & 58,59 & 125,80 \\
\hline Densidade de Comércios e Serviços ( $\mathrm{n}^{\circ}$ estabelecimentos $/ \mathrm{km}^{2}$ ), círculo $500 \mathrm{~m}$ & 1149,27 & 1621,98 & 47,11 & 6140,82 \\
\hline Frequência de ônibus ( $\mathrm{n}^{\circ}$ total de viagens das linhas que servem a área), círculo $500 \mathrm{~m}$ & 3387,34 & 3696,34 & 248 & 13823 \\
\hline Acidentes $\left(\mathrm{n}^{\circ}\right)$, círculo $500 \mathrm{~m}$ & 671,36 & 582,55 & 32 & 1957 \\
\hline \multicolumn{5}{|l|}{ Atitudinais } \\
\hline Pro-caminhada & 3,35 & 2,02 & $-0,25$ & 5,91 \\
\hline Pro-automóvel & 3,58 & 2,23 & $-0,56$ & 7,58 \\
\hline \multicolumn{5}{|l|}{ Afirmações atitudinais } \\
\hline Sempre que possível, eu prefiro caminhar em vez de dirigir & 6,46 & 3,55 & 0,00 & 10,00 \\
\hline Eu gosto de caminhar & 6,44 & 3,44 & 0,00 & 10,00 \\
\hline Caminhar, às vezes é mais fácil para mim do que dirigir & 5,96 & 3,86 & 0,00 & 10,00 \\
\hline Sempre que possível, prefiro utilizar transporte público ao invés de dirigir & 4,65 & 3,78 & 0,00 & 10,00 \\
\hline Eu gosto de utilizar transporte público & 4,02 & 3,37 & 0,00 & 10,00 \\
\hline Andar de carro é mais seguro contra assaltos e/ou roubos do que caminhar & 6,41 & 2,92 & 0,00 & 10,00 \\
\hline $\begin{array}{l}\text { Andar de carro é mais seguro contra assaltos e/ou roubos do que utilizar transportes } \\
\text { público }\end{array}$ & 6,19 & 3,06 & 0,00 & 10,00 \\
\hline Eu gosto de dirigir & 5,02 & 3,97 & 0,00 & 10,00 \\
\hline Preciso de carro para realizar as minhas atividades do dia-a-dia & 5,04 & 3,93 & 0,00 & 10,00 \\
\hline \multicolumn{5}{|l|}{ Percepção de segurança pública e de trânsito } \\
\hline Meu bairro é seguro (contra roubos e assaltos) para caminhar durante o dia & 7,56 & 2,78 & 0,00 & 10,00 \\
\hline Meu bairro é seguro (contra roubos e assaltos) para caminhar durante a noite & 2,41 & 2,76 & 0,00 & 10,00 \\
\hline O risco de acidentes de tráfego no meu bairro é pequeno & 5,81 & 2,91 & 0,00 & 10,00 \\
\hline
\end{tabular}

com o encontrado por Larrañaga et al. (2014) para Porto Alegre e por Cervero et al. (2009) para a cidade de Bogotá.

A variável Declividade mostrou ser um preditor significativo na decisão de realizar viagens a pé. A topografia da cidade apresenta aclives e declives que dificultam os deslocamentos não motorizados. Aclives acentuados desestimulam a caminhada. Esse resultado coincide com o encontrado por Larrañaga et al. (2014) para Porto Alegre.

Em relação às variáveis socioeconômicas, a variável Idade apresentou sinal negativo, indicando que pessoas mais jovens tendem a realizar mais viagens a pé. As variáveis Renda Média e Renda Alta, relacionadas à renda familiar mensal, apresentaram também sinal negativo. À medida que aumenta o rendimento familiar diminui a probabilidade da decisão pela caminhada. As variáveis atitudinais Pró-caminhada e Pró-automóvel foram significativas, indicando a importância de incluir variáveis que caracterizem o estilo de vida dos indivíduos, convicções pessoais e predisposições em relação aos modos de transporte. O sinal positivo da variável Pró-caminhada mostra que indivíduos com maior predisposição a caminhar, efetivamente caminham mais do que indivíduos com menor predisposição a usar este modo. Por outro lado, o sinal negativo da variável Próautomóvel mostra que indivíduos com maior predisposição a utilizar automóvel, efetivamente caminham menos do que indivíduos com menor predisposição a usar este modo.

A comparação do impacto das variáveis explicativas na decisão de caminhar foi realizada através do cálculo da elasticidade. A Tabela 4 apresenta as elasticidades da probabilidade da decisão de caminhar em relação às variáveis explicativas computadas e as elasticidades calculadas por Larranaga et al. (2014) para frequência de viagens a pé.

$\mathrm{O}$ valor de elasticidade com respeito à Densidade $\mathrm{Po}$ pulacional é de 0,35. Este valor é sensivelmente superior ao obtido na modelagem de frequência de viagens a pé em Porto Alegre, que correspondeu a 0,09. O valor de elasticidade para esta variável obtida em outros estudos varia entre 0,01 e 1,39, e o valor médio calculado é de 0,07 (Ewing e Cervero, 2010). O efeito da densidade populacional na decisão de realizar viagens é significativamente mais alto do 
Tabela 3. Modelo logit binomial para decisão de realizar viagem a pé

\begin{tabular}{|c|c|c|c|c|}
\hline & \multicolumn{2}{|c|}{ Logit binomial } & \multicolumn{2}{|c|}{ Logit misto $E C$} \\
\hline Variáveis & Coeficientes & Valor-p & Coeficientes & Valor-p \\
\hline \multicolumn{5}{|l|}{ Viagem e socioeconômicas } \\
\hline Idade (anos) & $-0,018$ & 0,000 & $-0,017$ & 0,000 \\
\hline Renda Média $(1,0)$ & $-0,500$ & 0,010 & $-0,404$ & 0,060 \\
\hline Renda Alta $(1,0)$ & $-0,903$ & 0,000 & $-1,060$ & 0,000 \\
\hline \multicolumn{5}{|l|}{ Estrutura urbana } \\
\hline Densidade Populacional, 500m & $6,66 \mathrm{E}-05$ & 0,000 & $4,53 \mathrm{E}-05$ & 0,010 \\
\hline \multicolumn{5}{|l|}{ Densidade de Interseções 4 aproximações, 500m } \\
\hline Declividade, $500 m$ & $-8,390$ & 0,010 & $-4,570$ & 0,010 \\
\hline \multicolumn{5}{|l|}{ Densidade Comércios e Serviços, 500m } \\
\hline \multicolumn{5}{|l|}{ Predisposições de viagem } \\
\hline Pro-caminhada_tp & 0,089 & 0,050 & 0,105 & 0,020 \\
\hline Pro-automóvel & $-0,098$ & 0,020 & $-0,112$ & 0,010 \\
\hline Constante & 0,773 & 0,007 & 0,058 & 0,200 \\
\hline \multirow[t]{2}{*}{ Sigma } & \multicolumn{2}{|c|}{-} & 0,296 & 0,100 \\
\hline & \multicolumn{2}{|c|}{$\begin{array}{l}\text { Número de observações }=884 \\
\text { Log likelihood }=-509,388 \\
\text { Pseudo-R }{ }^{2}=0,151\end{array}$} & \multicolumn{2}{|c|}{$\begin{array}{l}\text { Número de observações }=884 \\
\text { Log likelihood=-501,984 } \\
\text { Pseudo-R }{ }^{2}=0,163\end{array}$} \\
\hline
\end{tabular}

\begin{tabular}{lcc}
\multicolumn{2}{c}{ Tabela 4. Elasticidades da decisão de caminhar } \\
\hline Variáveis & Frequência de viagens a pé & Decisão de realizar viagens a pé \\
\hline Socioeconômicas & & \\
\hline Idade (anos) & $-0,11$ & $-0,50$ \\
Renda média (1,0) & $-0,01$ & $-0,05$ \\
Renda alta (1,0) & $-0,05$ & $-0,30$ \\
\hline Estrutura urbana & 0,09 & 0,35 \\
\hline Densidade populacional, 500m & 0,08 & 0,34 \\
Densidade de interseções de 4 aproximações, 500m & $-0,03$ & $-0,11$ \\
Declividade média, 500m & 0,02 & 0,06 \\
Densidade de comércios e serviços, 500m &
\end{tabular}

que o efeito no número de viagens a pé em Porto Alegre. O impacto desta variável em Porto Alegre parece ser superior ao obtido em outros estudos.

A elasticidade calculada para o Densidade de interseções de 4 aproximações é de 0,34, mostrando que, juntamente com a Densidade populacional, é uma das variáveis de ambiente construído, com maior impacto na decisão de caminhar. O resultado sobre as variáveis mais influentes é semelhante ao encontrado na modelagem de frequência de "viagens a pé". Entretanto, o valor de elasticidade obtido para aquela variável na decisão de caminhar é sensivelmente superior ao obtido na modelagem de frequência (elasticidade da frequência de viagens a pé $=0,08$ ). $\mathrm{O}$ valor de elasticidade para esta variável obtida em outros estudos varia entre 0,00 e 1,11 , e o valor médio calculado é de 0,39 (Ewing e Cervero, 2010).

O efeito da Declividade média é de - 0,11 . O cálculo do impacto desta variável é uma referência muito útil para planejadores urbanos, pois raramente é considerada em outros estudos. Na modelagem de frequência de viagens a pé realizada em Porto Alegre o valor obtido foi de $-0,03$, inferior ao computado neste caso.

A elasticidade calculada para o Densidade de comércios e serviços é de 0,06 . Este valor também é superior ao calculado para frequência de viagens a pé. Não foram encontrados valores de elasticidade sobre esta variável na literatura. Elasticidades de variáveis relacionadas com presença de comércios e serviços variam entre 0,02 e 0,25 (Ewing e Cervero, 2010).

\section{CONSIDERAÇÕES FINAIS}

O estudo realizado fornece evidências que, na cidade de Porto Alegre, a estrutura urbana influencia a decisão de realizar viagens a pé. Os resultados obtidos permitem concluir que bairros densos, com padrão viário em forma de grelha, topografia pouco acentuada e comércios e serviços próximos à residência estimulam a decisão de caminhar na cidade de Porto Alegre. Os resultados obtidos apoiam a hipótese adotada a priori, na qual se esperava replicar muitos dos resultados já relatados na literatura.

No entanto, obtiveram-se algumas diferenças nas magnitudes do impacto de algumas variáveis, impactos que foram medidos como elasticidades. Os resultados das elasticidades calculadas para a decisão de caminhar com relação às variáveis da estrutura urbana foram maiores do que em outros estudos reportados na literatura. Comparando com os resultados obtidos na modelagem de frequência de viagens a pé para Porto Alegre, as elasticidades calculadas para a decisão de caminhar também foram maiores das obtidas na modelagem de frequência. Assim, as variáveis urbanas impactam mais na escolha de caminhar ou não caminhar, do que na quantidade caminhada. Os resultados mostram que tanto frequência de viagens a pé, quanto escolha do modo a pé dependem com a mesma intensidade de características socioeconômicas e urbanas.

As elasticidades estimadas para todas as variáveis podem ser úteis para os planejadores. Os valores poderiam ser 
usados para Porto Alegre, ou para aplicações em lugares próximos à cidade ou com características similares. Estas elasticidades podem ser usadas no planejamento para calcular estimativas de caminhada em relação ao caso base, ou em previsões de modelos de demanda, para refletir a influência do ambiente construído.

Futuros estudos poderiam abordar o desenvolvimento de modelos híbridos de escolha mais complexos para explicar outras escolhas marginais de transporte (por exemplo, a decisão de possuir um carro). Também o desenvolvimento de modelos discretos-contínuos para modelar simultaneamente a distância caminhada em conjunto com a decisão de caminhar poderiam ser de grande contribuição.

\section{AGRADECIMENTOS}

Os autores agradecem ao CNPQ pelo apoio através da concessão de bolsa de pesquisa.

\section{REFERÊNCIAS}

Amâncio, M. A. (2005) Relacionamento entre a forma urbana e as viagens a pé. Dissertação de Mestrado. Departamento de Engenharia Civil, Universidade Federal de São Carlos, São Paulo.

Baran, P.; D. Rodríguez, e A. Khattak (2008) Space Syntax and Walking in a New Urbanist and Suburban Neighborhoods. Journal of Urban Design, v. 13, n. 1, p. 5-28. DOI: $10.1080 / 13574800701803498$

Ben-Akiva, M. e S. R. Lerman, (1985). Discrete choice analysis - Theory and applications to travel demand. MIT Press, Cambridge.

Ben-Akiva, M.E e D. Bolduc, (1996) Multinomial probit with a logit kernel and a general parametric specification of the covariance structure. Working Paper, Economique, Université Laval, Québec.

Ben-Akiva, M.; D. Bolduc e J. Walker (2003) Specification, Identification, and Estimation of the Logit Kernel (or Continous Mixed Logit) Model. Working Paper apresentado no 5th Invitational Choice Symposium, Asilomar, California.

Bierlaire, M. (2003) BIOGEME - A free package for the estimation of discrete choice models, 3rd Swiss Transportation Research Conference, Switzerland.

Boarnet, M. G.; M. Greenwald e T. McMillan (2008) Walking, urban design, and health: Toward a cost-benefit analysis framework. Journal of Planning Education and Research, v. 27, n. 3, p. 341-358. DOI: 10.1177/0739456X07311073

Brownstone, D.; K. E. Train (1999) Forescating new product penetration with flexible substitution patterns. Journal of Econometrics, v. 89, p. 109-129.

Cao, X.; Handy, S. e P. Mokhtarian (2006). The Influences of the Built Environment and Residential Self-Selection on Pedestrian Behavior: Evidence from Austin, TX. Transportation, v. 33, n. 1, p.1-20. DOI: 10.1007/s11116-005-7027-2

Cervero, R. e K. Kockelman (1997) Travel demand and the 3Ds Density, diversity, and design. Transportation Research D, v. 2, n. 3, p. 199-219. DOI: 10.1016/S1361-9209(97)00009-6
Cervero, R. e M. Duncan (2003) Walking, bicycling, and urban landscapes - evidence from San Francisco Bay Area. American Journal of Public Health, v. 93, n. 9, p. 1478-1483.

DOI: 10.2105/AJPH.93.9.1478

Cervero, R.; O. Sarmiento; E. Jacoby; L.F. Gomez e A. Neiman (2009). Influences of Built Environments on Walking and Cycling-Lessons from Bogotá. International Journal of Sustainable Transportation, v. 3, n. 4, p. 203- 226. DOI: $10.1080 / 15568310802178314$

Chatman, D. G (2009) Residential self-selection, the built environment, and nonwork travel - Evidence using new data and methods. Environment and Planning A, v. 41, n. 5, p. 1072-1089. DOI: $10.1068 / \mathrm{a} 4114$

Deus, L. R. (2008) A influência da forma urbana no comportamento de viagem das pessoas: Estudo de caso em Uberlândia, MG. Dissertação de Mestrado. Departamento de Engenharia Civil, Universidade Federal de São Carlos, São Paulo.

Domencich, T.; D. Mcfadden (1972) A disaggregated Behavioral Model of Urban Travel Demand. Report $N^{\circ}$ CRA-156-2, Charles River Associates, Inc. Cambridge, Massachusetts.

Ewing, R. e R. Cervero (2001) Travel and the Built Environment - A Synthesis. Transportation Research Record - Journal of the Transportation Research Board 1780, p. 87-114. DOI: $10.3141 / 1780-10$

Ewing, R.; M. J. Greenwald; M. Zhang; J. Walters; M. Feldman; R. Cervero e J. Thomas (2009). Measuring the impact of urban form and transit access on mixed use site trip generation rates Portland pilot study. DC: U.S. Environmental Protection Agency, Washington.

Ewing, R. e R. Cervero (2010) Travel and the Built Environment - A Meta-Analysis. Journal of the American Planning Association, v. 76, n. 3, p. $265-294$.

DOI: $10.1080 / 01944361003766766$

Fernandes, K. D.; M. L. Maia; C. Ferraz (2008) Forma urbana e deslocamentos pendulares: análise dos bairros de Casa Caiada e Jardim Brasil em Olinda/PE. In: XXII ANPET Congresso de Pesquisa e Ensino em Transportes. Fortaleza.

Frank, L. D. e P. O. Engelke (2001) The Built Environment and Human Activity Patterns - Exploring the Impacts of Urban Form on Public Health. Journal of Planning Literature, v. 16, p. $202-$ 218. DOI: $10.1177 / 08854120122093339$

Frank, L. D.; S. Kavage; M. Greenwald; J. Chapman e M. Bradleyn (2009) I-PLACE3S health \& climate enhancements and their application in King County. King County Health Scape, Seattle, WA.

Handy, S. L.; M. G. Boarnet; R. Ewing e R. E. Killingsworth (2002) How the built environment affects physical activity. Views from Urban Planning. American Journal of Preventive Medicine, 23(2). p. 64-73. DOI:10.1016/S0749-3797(02)00475-0

Larranaga, A. M. L. (2012) Estrutura urbana e viagens a pé. Tese de Doutorado. Programa de Pós-Graduação em Engenharia da Produção, Universidade Federal do Rio Grande do Sul, Porto Alegre.

Larranaga, A. M. L.; L. I. Rizzi; J. Arellana; O. Stramb e H. Cybis (2014) The Influence of built environment and travel 
attitudes on walking: a case study of Porto Alegre, Brazil. International Journal of Sustainable Transportation. DOI:10.1080/15568318.2014.933986

Lee, C. e A. V. Moudon (2006) Correlates of Walking for Transportation or Recreation Purposes. Journal of Physical Activity and Health, v. 3, n. S1, p. S77-S98.

Manski, C. (1977). The structure of random utility models. In: Theory and Decision, v. 8, issue 3, p. 229-254.

DOI: $10.1007 / \mathrm{BF} 00133443$

Mcfadden, D. (1974). Conditional logit analysis of qualitative choice behavior. In: P Zarembka (ed.) Frontiers in Econometrics. Academic Press, New York.

Mcfadden, D.; K. Train (2000) Mixed MNL Models for Discrete Response. Journal of Applied Econometrics, sep/oct., v. 15, issue 5, p. 447-470, John Wiley \& Sons, Ltd., New York. DOI: $10.1002 / 1099-1255(200009 / 10) 15: 5<447$

AID-JAE570>3.0.CO;2-1

Naess, P. (2005) Residential location affects travel behavior - but how and why? The case of Copenhagen metropolitan area.

Progress in Planning, v. 63, issue 2, p. 167-257.

DOI: $10.1016 /$ j.progress.2004.07.004

Ortúzar, J. D. e L. G. Willumsen. (2011) Modelling Transport. 3 th ed. Wiley, Chichester.

Sehatzadeh, B.; R. B. Noland e M. D. Weiner (2011) Walking frequency, cars, dogs, and the built environment. Transportation Research Part A, v. 45, p. 741-754.

DOI:10.1016/j.tra.2011.06.001

Wewers, M. E. e N. K. Lowe (1990). A critical review of visual analogue scales in the measurement of clinical phenomena. Research in Nursing and Health, v. 13, p. 227-236.

DOI:10.1002/nur.4770130405

Zegras, C. (2004) The Influence of Land Use on Travel Behavior - Empirical Evidence from Santiago de Chile. TRB 2004 Annual Meeting of the Transportation Research Board - Travel Demand and Land Use. 83th Annual Meeting, Washington.

Zegras, C. (2010) The Built Environment and Motor Vehicle Ownership and Use - Evidence from Santiago de Chile. Urban Studies, v. 47, n. 8.

DOI: $10.1177 / 0042098009356125$ 